\title{
Evaluation of a low cost high fidelity arterial simulation device for ultrasound guided vascular puncture and cannulation.
}

K. Bull, J. Willers, R. Jeevananthan, J. Macallan, C. Long, N. Duke

Department of Anaesthesia and Intensive Care, Worthing Hospital, Lyndhurst Road, Worthing, West Sussex, United Kingdom

\section{Introduction}

Obtaining ultrasound guided arterial access is a desirable skill for the modern day anaesthetist. Vascular access phantoms incorporating mechanical pump devices simulating pulsation for arterial puncture and cannulation are available, but costly. We endeavoured to create a low cost, effective, high fidelity simulation device and evaluate it for teaching ultrasound guided arterial cannulation. We developed a fully functional, high fidelity arterial system compatible with various procedures.

\section{Method}

An existing variable volume/pressure arterial simulation pump [1] was connected to an elastic artery analogue (160Q size modelling balloon). This was connected to a pigmented fluid source at diastolic pressure (Fig 1). The pulsations at 60 per minute, and systolic pressure were provided by manipulating pump settings suiting the compliance of the vessel model. Any fluid aspirated from the system was automatically replaced at diastolic pressure. The arterial analogue was placed between two layers of ADAM gel (Aqueous Dietary fibre Antifreeze Mix gel) an ultrasound medium resistant to needle tract damage (Fig 2). The total cost was $<£ 40$, of which $£ 30$ was for the pump. It was then evaluated as a training tool using an established assessment questionnaire.
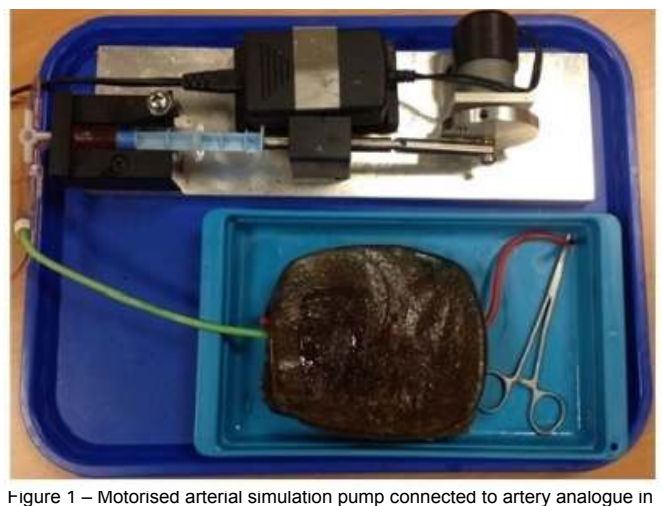
ADAM gel

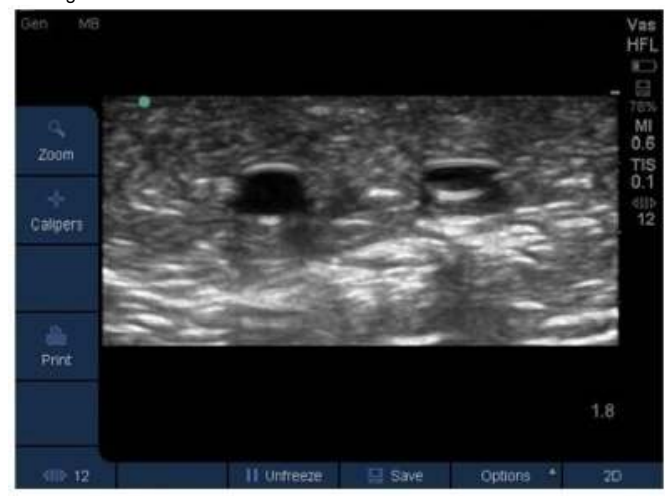

Figure 2 - Static ultrasound image taken at the end of the study to show artery with pump driven pulsations (left) and adjacent compressible vein (right) to enable arterial cannulation

\section{References}

Bukunola, L. Bisht Presented at AAGBI WSM 2015

2. Guidelines, AAGBI: Safer vascular access 2016. A. Bodenham (Chair), S. Babu, J. Bennett, R. Binks, P. Fee, B. Fox, A.J. Johnston, A.A. Klein, J.A. Langton, H. Mclure and S.Q.M. Tighe

\section{Results}

The evaluation from 15 Anaesthetists at various stages of training is summarised below (Fig 3 ) along with a photo of the phantom being used for arterial cannulation (Fig 4).

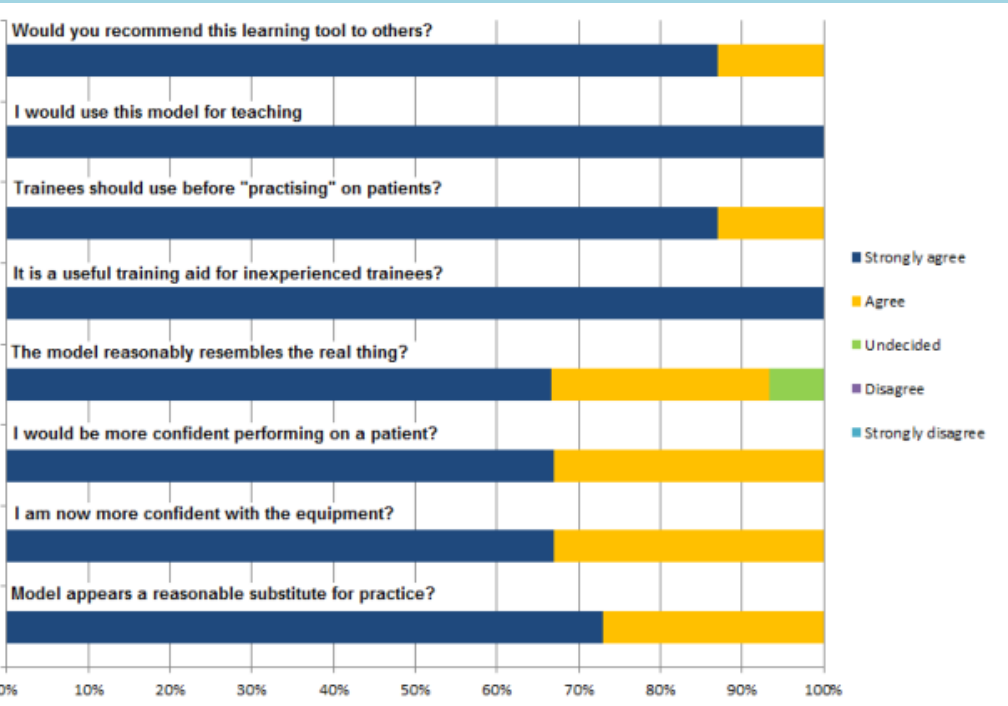

Figure 3 - Bar chart to show results of evaluation of high fidelity arterial simulation device as a training too

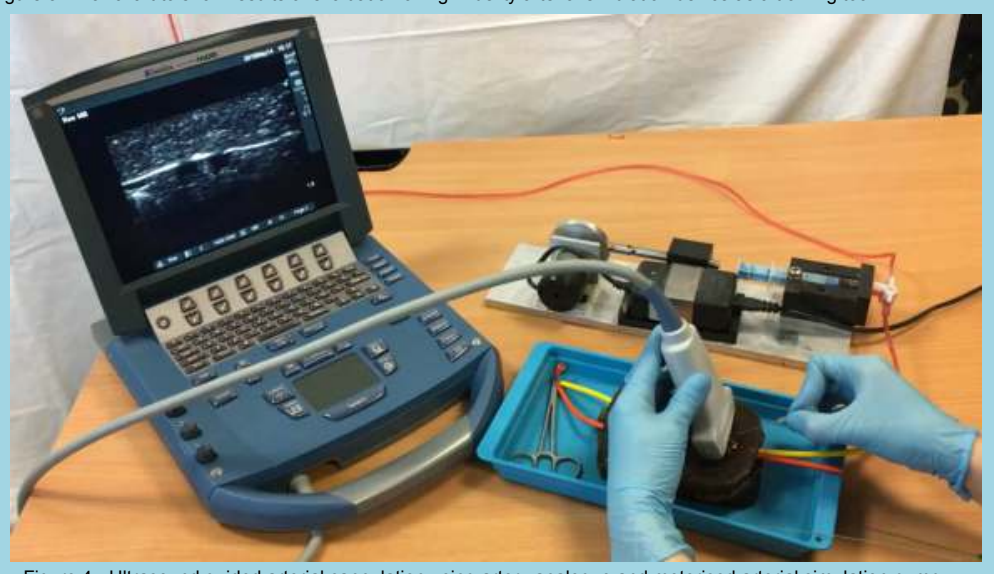

\section{Discussion}

The experienced trainees (ST3 - Associate Specialist) surveyed commented that our arterial simulation device provided 'a realistic ultrasound image and a similar experience to using ultrasound for arterial cannulation on real patients'. Two novice anaesthetic trainees without prior experience of arterial cannulation concluded that it was a 'good training model'. $100 \%$ of those surveyed strongly agreed that the model was a useful training aid for inexperienced trainees and they would use this model for teaching.

\section{Conclusion}

It is possible to construct a high fidelity arterial access simulation phantom device at a reasonable cost that functions as an effective training tool. Our device is particularly relevant in light of the 2016 AAGBI Safer vascular access guidelines [2], which state that real time ultrasound should be used early for difficult arterial cannulation and to increase the safety and success of procedures.

Leading on from this we intend to broaden the use of this phantom by incorporating ultrasound guided venous cannulation and evaluating this as a teaching aid . In addition, to practice more difficult vascular access, this model could be modified to simulate vessels at variable depths. 